\title{
Universiteit
}

Leiden

The Netherlands

\section{Bulk and Surface Nucleation Processes in Ag2S Conductance Switches}

Morales Masis, M.; Molen, S.J. van der; Hasegawa, T.; Ruitenbeek, J.M. van

\section{Citation}

Morales Masis, M., Molen, S. J. van der, Hasegawa, T., \& Ruitenbeek, J. M. van. (2011). Bulk and Surface Nucleation Processes in Ag2S Conductance Switches. Physical Review B, 84(11), 115310. doi:10.1103/PhysRevB.84.115310

Version: $\quad$ Not Applicable (or Unknown)

License: $\quad$ Leiden University Non-exclusive license

Downloaded from: https://hdl.handle.net/1887/61265

Note: To cite this publication please use the final published version (if applicable). 


\title{
Bulk and surface nucleation processes in $\operatorname{Ag}_{2} \mathrm{~S}$ conductance switches
}

\author{
M. Morales-Masis, ${ }^{1, *}$ S. J. van der Molen, ${ }^{1}$ T. Hasegawa, ${ }^{2}$ and J. M. van Ruitenbeek ${ }^{1}$ \\ ${ }^{1}$ Kamerlingh Onnes Laboratorium, Universiteit Leiden, P.O. Box 9504, NL-2300 RA Leiden, The Netherlands \\ ${ }^{2}$ WPI Center for Materials Nanoarchitectonics, National Institute for Materials Science, 1-1 Namiki, Tsukuba, \\ Ibaraki 305-0044, Japan
}

(Received 6 July 2011; published 19 September 2011)

\begin{abstract}
We studied metallic Ag formation inside and on the surface of $\mathrm{Ag}_{2} \mathrm{~S}$ thin films, induced by the electric field created with a scanning tunnel microscope (STM) tip. Two clear regimes were observed: cluster formation on the surface at low bias voltages, and full conductance switching at higher bias voltages $(V>70 \mathrm{mV})$. The bias voltage at which this transition is observed is in agreement with the known threshold voltage for conductance switching at room temperature. We propose a model for the cluster formation at low bias voltage. Scaling of the measured data with the proposed model indicates that the process takes place near steady state, but depends on the STM tip geometry. The growth of the clusters is confirmed by tip retraction measurements and topography scans. This study provides improved understanding of the physical mechanisms that drive conductance switching in solid electrolyte memristive devices.
\end{abstract}

DOI: 10.1103/PhysRevB.84.115310

PACS number(s): 73.40.-c, 72.60.+g, 73.63.Rt

\section{INTRODUCTION}

The interest in studying metal cluster formation in chalcogenide materials comes from the prospect of building nanometer-scale conductance switches with the configuration metal/chalcogenide/metal (M/C/M). In this configuration, changes in the conductance are induced by the application of a voltage between the two metal electrodes. Depending on the chalcogenide material, the conductance switching is caused by phase transitions ${ }^{1,2}$ or ionic transport processes. $^{3-6}$

The amplitude of the voltage necessary to switch an $\mathrm{M} / \mathrm{C} / \mathrm{M}$ system from the low conductance state to the high conductance state has a threshold value $\left(V_{t h}\right)$. This value is the voltage at which the energy barrier for nucleation of a metallic phase inside the chalcogenide material is overcome. The threshold voltages are strongly dependent on the chalcogenide material, electrode material, and geometry of the metal-chalcogenidemetal cell. ${ }^{4,7-10}$

In the case of ionic transport, conductance switching is due to the formation and dissolution of metallic filaments between the electrodes. The formation and dissolution of such nanoscale metallic filaments is driven by the polarity and amplitude of the voltage applied. ${ }^{3,11}$ One of the distinguishing properties of ionic switches is the fact that the diffusion constant of ions at room temperature can be very large, which is an advantage in view of low energy operation (low voltages to switch on and off), but a disadvantage in view of a short memory retention time.

We focus on the study of conductance switching due to ion transport and we use $\mathrm{Ag}_{2} \mathrm{~S}$ as the model material. Our interest lies in the understanding of the physical processes that drive nucleation and filament formation, including threshold voltages and growth kinetics. Previously, we studied the transport processes within $\mathrm{Ag}_{2} \mathrm{~S}$ thin films, which occur under steady-state conditions, i.e., before switching. ${ }^{12}$ We used a conducting AFM tip brought in contact with the $\mathrm{Ag}_{2} \mathrm{~S}$ sample. For $\mathrm{Ag}$ nucleation inside the $\mathrm{Ag}_{2} \mathrm{~S}$ film giving full conductance switching, a critical supersaturation of $\mathrm{Ag}$ ions inside the
$\mathrm{Ag}_{2} \mathrm{~S}$ film is needed. This phenomenon only occurs at bias voltages above $70 \mathrm{mV},{ }^{24}$ which is defined as the threshold voltage for switching at room temperature. In this paper, we confirm experimentally this threshold voltage for nucleation inside the film. Furthermore, we show that when starting from a tunneling gap between the top electrode and the sample, Ag clusters can be grown between the tip and the $\mathrm{Ag}_{2} \mathrm{~S}$ surface at voltages significantly lower than the observed threshold voltage for switching. At voltages above the threshold, a rapid nucleation inside the $\mathrm{Ag}_{2} \mathrm{~S}$ thin film is observed and the sample then exhibits full conductance switching. The two processes can be separated by properly preparing the initial conditions.

\section{EXPERIMENTAL PROCEDURE}

The samples consist of $\mathrm{Ag}(200 \mathrm{~nm}) / \mathrm{Ag}_{2} \mathrm{~S}$ (200 nm) layers deposited on a $\mathrm{Si}$ substrate. The $\mathrm{Ag}$ film is sputtered on a $\mathrm{Si}(100)$ substrate covered with a native oxide layer. On top of the $\mathrm{Ag}$ layer, the $\mathrm{Ag}_{2} \mathrm{~S}$ film is grown by sputtering of $\mathrm{Ag}$ in a $\mathrm{Ar} / \mathrm{H}_{2} \mathrm{~S}$ plasma. The sample preparation is described in more detail in Ref. 12. The surface roughness of the sample is approximately $30 \mathrm{~nm}$.

As the top electrode, we use a scanning tunnel microscope (STM) tip which is manually cut from either a Pt or PtIr wire. The measurements are performed at room temperature in a JEOL UHV STM/AFM system with a base pressure of $1 \times 10^{-9}$ mbar. In this STM setup, the tunneling voltage is applied to the sample while the tip is connected to ground.

For the measurements we have connected an external data acquisition (DAQ) card from National Instruments to the JEOL STM controller. Our measurement software allows the simultaneous control of two outputs (bias voltage, piezovoltage) and the monitoring and recording of two inputs (current and $Z$ piezovoltage). Hence, the measurements are fully controlled through LABVIEW, independent of the STM controller. 


\section{CONDITIONS FOR VACUUM TUNNELING ON $\mathrm{Ag}_{2} \mathrm{~S}$}

When performing STM measurements on $\mathrm{Ag}_{2} \mathrm{~S}$, two important points must be considered: (1) $\mathrm{Ag}_{2} \mathrm{~S}$ is an $n$-type semiconductor, with a band gap ranging between 0.6 and $1.2 \mathrm{eV} .^{13,14}$ (2) $\mathrm{Ag}_{2} \mathrm{~S}$ has both ionic and electronic mobile charges. The ionic mobile charges $\left(\mathrm{Ag}^{+}\right.$ions) act as $n$-type dopants within the $\mathrm{Ag}_{2} \mathrm{~S}$ film. Therefore, a local accumulation of ions in the sample causes changes in the local conductivity and band gap of the film. In our experiments, we apply a voltage between the STM tip (top contact) and the Ag layer (bottom contact), creating a strongly localized electric field near the tip. If a positive sample bias is applied, the mobile $\mathrm{Ag}^{+}$ions in the $\mathrm{Ag}_{2} \mathrm{~S}$ will move toward the region closest to the tip, increasing the local conductivity and lowering the band gap. Due to the points mentioned above, the apparent sample resistance can vary depending on the values of the tunneling gaps and applied voltages.

We measured IV curves to characterize our sample and confirm the characteristics of the band gap and the effect of the mobile ions. A typical IV curve, measured at the tunneling gap of $10 \mathrm{G} \Omega$, is shown in Fig. 1(a). The IV curve is asymmetric, with a flat region between approximately $-600 \mathrm{mV}$ and $+400 \mathrm{mV}$. This flat region is the result of the band gap of the sample. At the negative bias the rapid increase in conductance below $-600 \mathrm{mV}$ is associated with the valence-band edge. At the positive bias side above $+400 \mathrm{mV}$ the rise of the curve is related to the position of the conduction band edge. The position of the conduction band edge can be influenced by the $n$-type doping due to the accumulation of $\mathrm{Ag}^{+}$ions.

The effects of $n$-type doping become more important when the tunneling gap is reduced. When working at tunneling gaps with a resistance in the range of several megohms, a large part of the voltage drops across the sample (the sample resistance for a $\mathrm{Pt}$ atomic point contact with the $\mathrm{Ag}_{2} \mathrm{~S}$ sample is $>3 \mathrm{G} \Omega$ for voltages below the band edge), and therefore the activation of ions to move toward the tip is higher than for large tunneling gaps. Figure 1(b) presents an IV curve measured at a tunneling gap of $25 \mathrm{M} \Omega$, set at a bias of $-1 \mathrm{~V}$. The $I V$ curve shows an exponential increase in the current at the positive bias side,
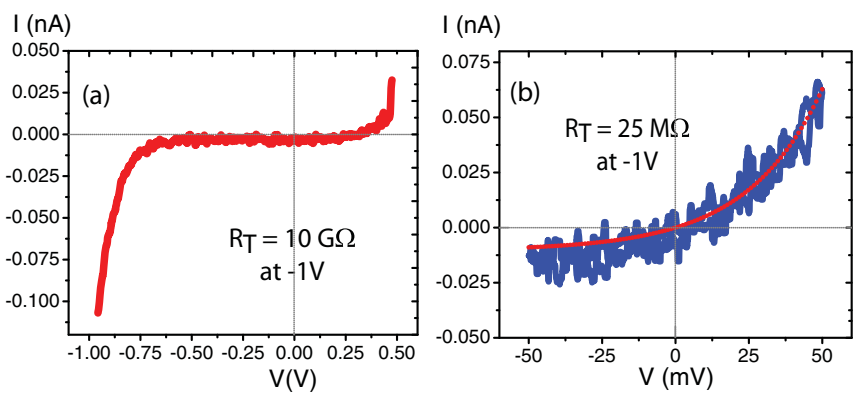

FIG. 1. (Color online) Current-voltage characteristics of the $\mathrm{Ag}_{2} \mathrm{~S}$ thin film at two different tunneling gaps. (a) At $10 \mathrm{G} \Omega$ (tunneling gap defined at $-1 \mathrm{~V}$ ), the $I V$ curve indicates the presence of the band gap in the $\mathrm{Ag}_{2} \mathrm{~S}$ sample. (b) At $25 \mathrm{M} \Omega$ (tunneling gap defined at $-1 \mathrm{~V}$ ), the $I V$ curve shows an exponential increase in the current at positive bias, due to the accumulation of $\mathrm{Ag}$ close to the tip for close proximity of the tip to the sample. The red curve is a fit of the $I V$ curve from which we calculate the effective top contact radius as $0.8 \mathrm{~nm}$.

at a voltage much lower than the band-gap edge. This $I V$ curve resembles our previous measurements ${ }^{12}$ and confirms the prediction by Hebb ${ }^{15}$ and Wagner, ${ }^{16}$ of an accumulation of $\mathrm{Ag}^{+}$ions resulting in an enhanced electronic conductivity and an asymmetric $I V$ curve with an exponential increase in the current at positive sample bias. Additionally, fitting the exponential IV curve with the Hebb-Wagner formalism [red curve in Fig. 1(b)], allows us to calculate the top contact radius. We obtained an effective contact radius of $0.8 \mathrm{~nm}$, indicating the close proximity, or touching, of the tip to the sample.

In order to assure that the tip is not in contact with the $\operatorname{Ag}_{2} \mathrm{~S}$ film, we choose to work with tunneling resistance between 10 and $1 \mathrm{G} \Omega$, which is set at a bias below the band edge at a voltage of $-1 \mathrm{~V}$. We use positive sample bias to activate the ion mobility and $\mathrm{Ag}$ growth.

\section{NUCLEATION AND CONDUCTANCE SWITCHING}

Our initial goal was to measure the growth rate of the $\mathrm{Ag}$ cluster (or filament), by following its growth with the STM tip, where the tip-sample (or tip-cluster) distance was kept constant by using the proper feedback parameters. ${ }^{17}$ Figure 2 is an example of such a measurement. For this specific trace, a tunneling gap is first defined at $-1 \mathrm{~V}$ sample bias and $0.3 \mathrm{nA}$ tunneling current. Two seconds after starting the measurement, the bias voltage is stepped from $-1 \mathrm{~V}$ to $+20 \mathrm{mV}$ (change in sign of the current in Fig. 2). Almost immediately, an increase in the voltage output of the $Z$ piezo is measured, which continues until the bias voltage is switched back to $-1 \mathrm{~V}$. The increase of the $Z$ piezovoltage indicates the total displacement of the tip away from the sample. In $10 \mathrm{~s}$ we measure a total displacement of $36 \mathrm{~nm}$. The tip displacement therefore indicates the growth of a protrusion on the surface of the sample. We expect this protrusion to be composed of $\mathrm{Ag}$ atoms, due to the reduction of $\mathrm{Ag}^{+}$ions at the surface of the $\mathrm{Ag}_{2} \mathrm{~S}$ film. ${ }^{17,18}$

Although qualitatively successful, this type of measurement has the disadvantage that tip contact with the sample cannot be avoided when the voltage is stepped from $-1 \mathrm{~V}$ (tunneling voltage) to the positive voltage to induce the filament growth. Because of the large resistance of the sample at low voltages, initially the voltage drops over the sample, not over the tunnel gap. The feedback responds to the resulting drop in the current by pushing the tip into contact with the sample [note the

(a)

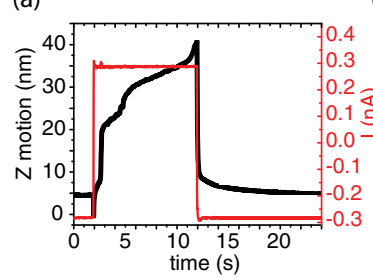

(b)
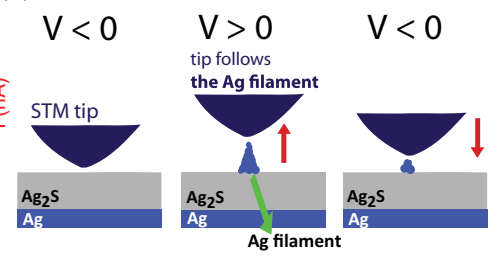

FIG. 2. (Color online) (a) $Z$ piezodisplacement (black curve) and tunneling current (red curve) as a function of time. The growth of the Ag cluster was activated with $+20 \mathrm{mV}$ bias. Keeping the feedback on allows us to follow the growth of the cluster in the $Z$ direction. (b) Schematic of the filament growth on the surface of the $\mathrm{Ag}_{2} \mathrm{~S}$ sample. 
sharp spike to a lower $Z$ position at the start in Fig. 2(a)]. At the same time, a filament starts growing. In many occasions the cluster grows much faster than the feedback speed of our STM, causing large oscillations in the $Z$ position and tunneling current. Because of this, the results show large variations in the growth rate, and it is not possible to accurately determine the dependence of the growth rate on the bias voltage.

As a different approach we decided to allow contact of the cluster with the tip by switching off the feedback of the STM and measuring the evolution of the current with time (instead of the $Z$ piezodisplacement). At the start of the measurements, the tunneling gap is fixed at $-1 \mathrm{~V}$ sample bias and $1 \mathrm{nA}$ tunneling current. With a tunneling gap thus defined, the feedback is switched off and a positive bias voltage is applied to the sample. The positive bias voltage is kept constant while the current is being recorded as a function of time.

Figure 3(a) presents a typical measurement. The measurement is performed as follows: at $t=0$ the feedback is switched off and we wait $2 \mathrm{~s}$ to confirm that the tip-sample distance remains constant. At $t=2 \mathrm{~s}$ the bias voltage $V$ is switched from $-1 \mathrm{~V}$ to $+50 \mathrm{mV}$. Immediately we observe a drop in the current, as expected from the high resistance of the sample at low bias voltages. A certain time elapses (induction time)
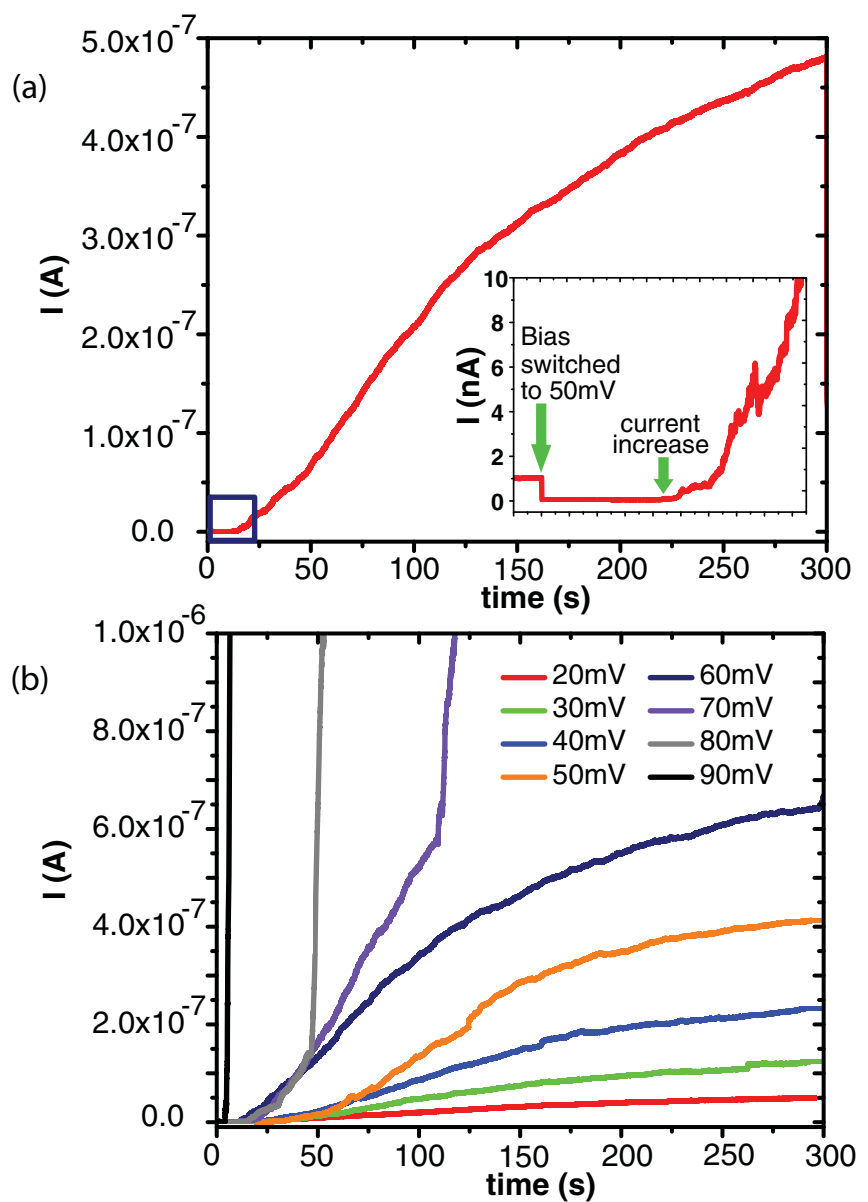

FIG. 3. (Color online) (a) Current evolution observed when a step in the voltage from $-1 \mathrm{~V}$ to $+50 \mathrm{mV}$ is applied to the sample. The inset shows the first seconds of the trace. (b) Traces measured at bias voltages from $+20 \mathrm{mV}$ up to $+90 \mathrm{mV}$. before the current starts increasing [see inset in Fig. 3(a)]. The current increases until the bias voltage is switched back to $-1 \mathrm{~V}$.

The same measurement was performed for bias voltages, $V$, ranging from +20 to $+90 \mathrm{mV}[$ Fig. 3(b)]. In this set of data, for the traces taken with $+20 \mathrm{mV}$ up to $+60 \mathrm{mV}$ we observe the same behavior as described for Fig. 3(a), with a systematic increase in the growth rate as a function of the applied bias voltage.

At $+70 \mathrm{mV}$ a particular curve is observed: during the first $100 \mathrm{~s}$ it resembles the previous traces. However, this behavior is interrupted by a sudden increase of the current growth rate. The current increase is such that it quickly saturates the current amplifier. A similar behavior is observed at $+80 \mathrm{mV}$, with the main difference that the strong increase in current is observed at an earlier time. At $+90 \mathrm{mV}$, the increase of the current occurs almost immediately after the positive voltage is applied.

The measurements presented in Fig. 3(b) suggest the presence of two distinct physical processes. Figure 4 presents further measurements performed in order to distinguish the two processes. Figure 4(a) presents a set of traces of conductance $(G)$ as a function of time, obtained by applying bias voltages of $+70 \mathrm{mV}$ and higher. With each run the sample conductance increases sharply, reaching values larger than $1 G_{0}$, where $G_{0}=2 e^{2} / h=77 \mu \mathrm{S}$ is the conductance quantum. We observe a systematic decrease of the induction time with bias. At $G>1 G_{0}$, the presence of a metallic Ag filament connecting (a)

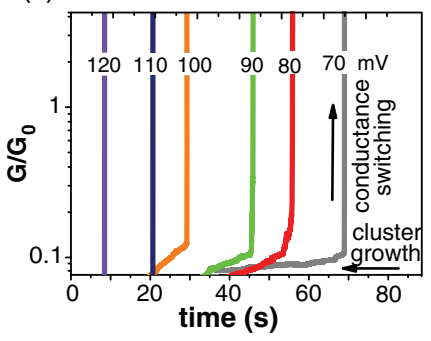

(c)

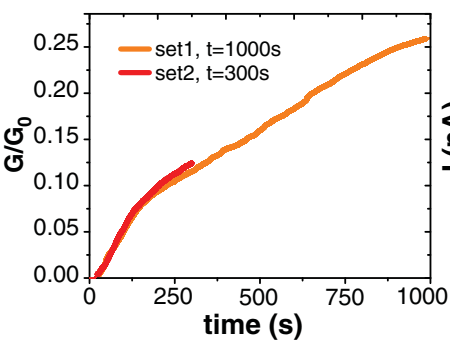

(b)

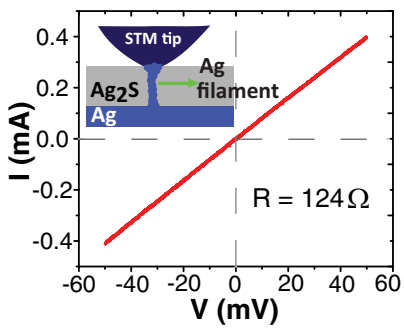

(d)

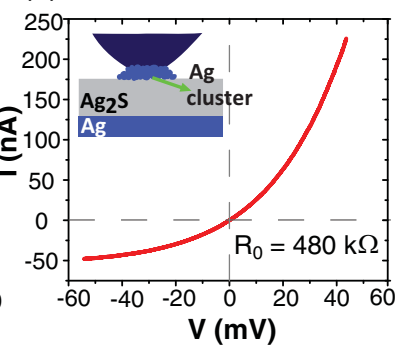

FIG. 4. (Color online) (a) Conductance switching induced at bias voltages $\geqslant+70 \mathrm{mV}$. The plot shows the increase of the conductance above $1 G_{0}$ and a systematic decrease in the induction time with bias. (b) IV curve measured in the high-conductance state of the sample, indicating metallic behavior. (c) Conductance trace measured for $1000 \mathrm{~s}$, applying a bias voltage of $+50 \mathrm{mV}$. The trace in red presents another trace measured at $+50 \mathrm{mV}$ for $300 \mathrm{~s}$. The trace does not show a sudden increase of the conductance for full switching. (d) $I V$ curve measured at $t=60 \mathrm{~s}$ of a trace measured with $+50 \mathrm{mV}$, demonstrating semiconducting behavior dominated by the bias-induced changes in ion concentration in the $\mathrm{Ag}_{2} \mathrm{~S}$ film (Ref. 12). 
the bottom electrode with the STM tip is expected. In the traces presented in Fig. 4(a), the final conductances (not shown in the plot) reach values as high as hundreds of $G_{0}$. In order to test for metallic conductance, we switch the sample to the high conductance state with $+110 \mathrm{mV}$ and measure an $I V$ curve [Fig. 4(b)]. The resulting curve is linear, with a slope indicating a resistance of $124 \Omega$ in this example. This confirms the metallic behavior of the sample which we attribute to the formation of a $\mathrm{Ag}$ filament inside the $\mathrm{Ag}_{2} \mathrm{~S}$ film.

To verify that the filament formation does not occur in the low voltage regime (from 0 to $+60 \mathrm{mV}$ ) we measured traces for times much longer than the 300 s typically used. Figure 4(c) shows one of the traces measured by applying a bias voltage of $+50 \mathrm{mV}$ for $1000 \mathrm{~s}$. The trace is plotted as the conductance $\left(G / G_{0}\right)$ vs time. It is clear from the figure that there is no sudden increase in the current and that the sample, even after $1000 \mathrm{~s}$, has a conductance lower than $1 G_{0}$, although it is not much smaller. At this point, we performed $I V$ measurements to probe the current-voltage behavior at this stage. Figure 4(b) presents the resulting nonlinear $I V$ curve, which confirms that the conductance is dominated by the dynamic doping behavior of the $\mathrm{Ag}_{2} \mathrm{~S}$ semiconductor.

Two processes are clearly observed from the behavior of the traces of current vs time: Ag surface nucleation, and $\mathrm{Ag}$ filament formation inside the $\mathrm{Ag}_{2} \mathrm{~S}$ film. At room temperature we demonstrate that this transition occurs at $70 \mathrm{mV}$. It is important to mention that this threshold voltage varies with temperature and with the electrode material used. We have used $\mathrm{Ag}$ as the bottom electrode, which fixes the chemical potential at the $\mathrm{Ag} / \mathrm{Ag}_{2} \mathrm{~S}$ interface ${ }^{19}$ and therefore all changes occur near the Pt tip. In previous studies, we observed that if we use Pt as the bottom as well as for the top contact, the threshold voltage to full conductance switching increases up to $200 \mathrm{mV} .{ }^{11}$

In the current vs time traces, the increase of the current is linked to the growth of a $\mathrm{Ag}$ cluster on the sample surface, giving rise to an increasing contact size to the $\mathrm{Ag}_{2} \mathrm{~S}$ film. Because the feedback of the STM is turned off when the positive bias voltage is applied, the tip does not change its position and the growth of a cluster will occur between the STM tip and the sample surface. As the gap between tip and surface is only of the order $1 \mathrm{~nm}$, the cluster will make contact with the STM tip at the very start of the process. As long as the positive bias is applied, the cluster will continue growing. The growth is expected to be in the radial direction, increasing the contact area with the $\mathrm{Ag}_{2} \mathrm{~S}$ surface and filling the volume between the tip and the surface. These two points are tested further in the following.

In order to confirm that the tip is indeed in contact with the cluster and that the cluster is metallic, we perform the measurement illustrated in Fig. 5(a).

For the measurement presented in Fig. 5(a), we formed a cluster by applying $+20 \mathrm{mV}$ sample bias. The current was allowed to increase to $1 \mathrm{nA}$, and then the tip was retracted at a speed of $4 \mathrm{~nm} / \mathrm{s}$. It takes approximately $16 \mathrm{~s}$ for the current to completely drop to the initial value. We observed that the drop of the current starts immediately upon tip retraction from the surface [see line at $t \approx 82 \mathrm{~s}$ in Fig. 5(a)]. When the cluster is metallic, the drop of the current should be related with a decrease of the cross-section area at the $\mathrm{Ag}_{2} \mathrm{~S} / \mathrm{Ag}$-cluster

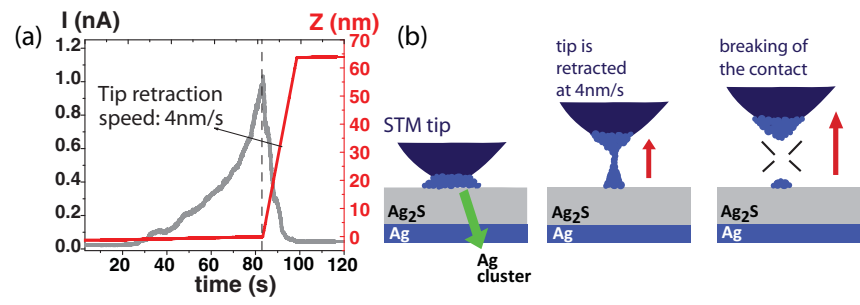

FIG. 5. (Color online) (a) Ag cluster formation induced at $+20 \mathrm{mV}$. When the tip current reaches $1 \mathrm{nA}$, the tip is retracted at a speed of $4 \mathrm{~nm} / \mathrm{s}$. As a consequence, the tip loses contact with the cluster and the current drops. (b) Schematic diagram of the cluster growth and neck formation when the tip is retracted.

interface. This occurs if the tip is dragging up a large part of the cluster when it is moving away from the surface, as illustrated by the cartoon in Fig. 5(b). Figure 5(a) shows that in $16 \mathrm{~s}$ the tip is retracted as much as $64 \mathrm{~nm}$ from the sample surface in the $Z$ direction. At much faster retraction rates, the time to break the contact with the cluster is considerably shorter. This experiment clearly shows that the cluster is in contact with the tip and that we can mechanically break this contact by retracting the tip over tens of nanometers.

\section{TOPOGRAPHY SCANS}

We scanned the surface of the $\mathrm{Ag}_{2} \mathrm{~S}$ film before and after the growth of a cluster (Fig. 6). For the scanning we used the tunneling parameters $-600 \mathrm{mV}$ and $1 \mathrm{nA}$. The sample was cooled down to $T \approx 240 \mathrm{~K}$ in order to reduce ion diffusion and prevent complete dissolution of the cluster before the scanning was performed.
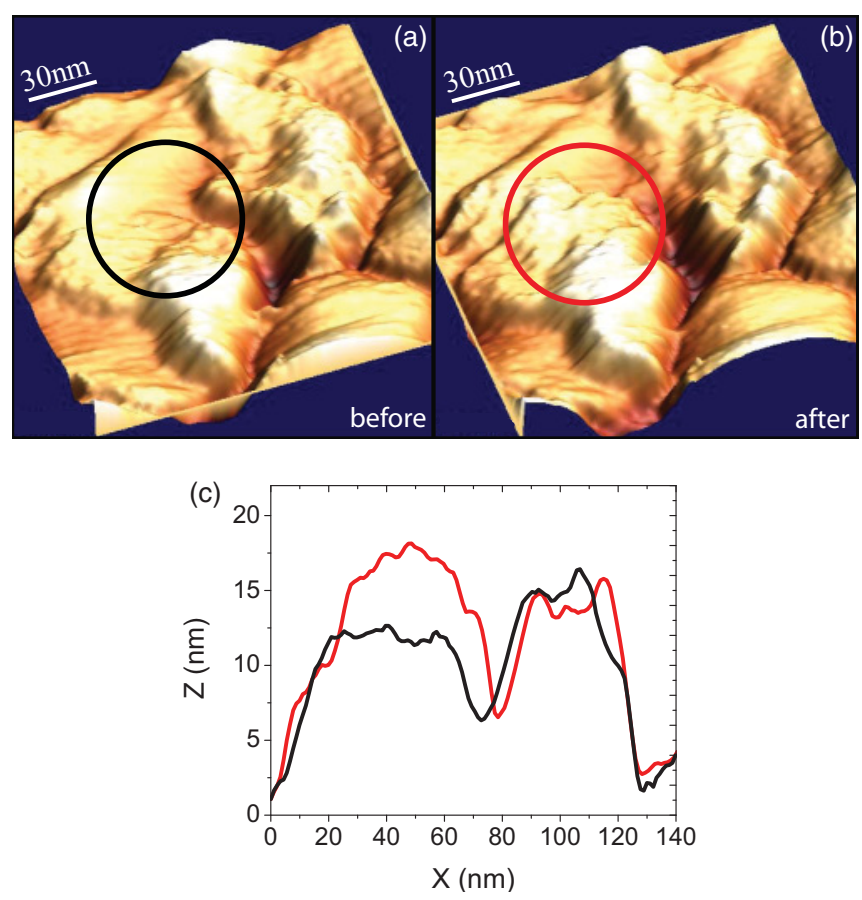

FIG. 6. (Color online) Topography scan (a) before and (b) after the growth of a cluster. (c) Line scan through the remnant of the grown cluster. 
We scanned an area of $250 \times 250 \mathrm{~nm}$ (in Fig. 6 we show only an area of $142 \times 142 \mathrm{~nm}$ ) and in the topography image we selected a flat spot on the sample to grow the cluster. The tip was placed above the selected position, and with the feedback on we applied a sample bias of $+300 \mathrm{mV}$. This large bias voltage will induce the growth of a large cluster, observed by the large displacement of the $Z$ piezo. This positive bias voltage was applied for $100 \mathrm{~s}$. After the $100 \mathrm{~s}$, the tunneling bias was set back $(-600 \mathrm{mV})$ and the tip restarted scanning the same area as before cluster growth.

Due to the large roughness of the surface of approximately $30 \mathrm{~nm}$, a slow scan speed needed to be used. The time taken from the moment the cluster was grown to the moment when the area of the cluster was scanned was more than $100 \mathrm{~s}$. At room temperature and with $-600 \mathrm{mV}$ sample bias the cluster is expected to be fully dissolved back in the $\operatorname{Ag}_{2} \mathrm{~S}$, as confirmed by our experiments. At $240 \mathrm{~K}$, we observed that after $100 \mathrm{~s}$, a small part of the cluster is still visible when the surface is imaged. A line scan through the remnants of the cluster [Fig. 6(c)], shows a height difference of $5 \mathrm{~nm}$, which is significantly smaller than the expected cluster height just after the switch (the measured displacement of the $Z$ piezo while performing the measurement was approximately $100 \mathrm{~nm}$ ). Nevertheless, the two distinct surface morphologies before and after the growth are clearly observed.

\section{DISCUSSION}

To understand the nucleation and the evolution of the cluster with time, we performed the following analysis. When a voltage $V$ is applied to the $\mathrm{Ag}_{2} \mathrm{~S}$ sample, between the $\mathrm{Ag}$ bottom contact and the Pt tip, the voltage drop over the sample $V_{s}$ imposes an electrochemical potential difference for the electrons at the two sample boundaries,

$$
-e V_{s}=\tilde{\mu}_{e}^{\prime \prime}-\tilde{\mu}_{e}^{\prime},
$$

with $-e$ the electron charge. Note that the total voltage applied drops partially over the tunneling gap and partially over the sample. Due to the large resistance of the sample, $V \approx V_{s}$.

The electrochemical potential of $\mathrm{Ag}^{+}$ions and electrons is related to the chemical potential of atoms by

$$
\mu_{\mathrm{Ag}}=\tilde{\mu}_{\mathrm{Ag}^{+}}+\tilde{\mu}_{e} .
$$

Then, Eq. (1) can also be written as

$$
-e V_{s}=\left(\mu_{\mathrm{Ag}}^{\prime \prime}-\mu_{\mathrm{Ag}}^{\prime}\right)-\left(\tilde{\mu}_{\mathrm{Ag}^{+}}^{\prime \prime}-\tilde{\mu}_{\mathrm{Ag}^{+}}^{\prime}\right) .
$$

Initially, the $\mathrm{Ag}^{+}$ions and electrons are distributed uniformly in the sample, thus $\nabla \mu_{\mathrm{Ag}}=0$. When the voltage is switched on, the gradients of the electrochemical potentials of electrons and $\mathrm{Ag}^{+}$ions will be identical in magnitude but opposite in sign. Opposing currents of ions $\left(j_{\mathrm{Ag}^{+}}\right)$and electrons $\left(j_{e}\right)$ are therefore set up in the sample.

When the top contact is an ion blocking contact (e.g., a Pt tip in contact with the $\mathrm{Ag}_{2} \mathrm{~S}$ sample), the silver ion current $\left(\mathrm{j}_{\mathrm{Ag}^{+}}\right)$ builds a concentration gradient at the $\mathrm{Ag}_{2} \mathrm{~S} / \mathrm{Pt}$-tip interface, until an equilibrium with the electrical potential gradient $\nabla \tilde{\mu}_{e}$ is reached, resulting in a steady state and a vanishing current of ions. Increasing the voltage further, increases the Ag concentration gradient, until the supersaturation reaches a critical value and metallic $\mathrm{Ag}$ is nucleated inside the $\mathrm{Ag}_{2} \mathrm{~S}$. This will occur at $V_{s}>70 \mathrm{mV}$ as demonstrated previously. ${ }^{12,20}$

Contrary to a blocking contact, in the present experiment there is initially a tunneling gap between the tip and the sample surface. Therefore ions are not fully blocked at the $\mathrm{Ag}_{2} \mathrm{~S} /$ vacuum interface, and surface nucleation is allowed. Anticipating that the surface nucleation energy is lower than the bulk nucleation energy, a Ag nucleus can be formed at the surface of the $\mathrm{Ag}_{2} \mathrm{~S}$ film at lower $\mathrm{Ag}$ saturation. The nucleus is produced by $\mathrm{Ag}^{+}$ions that are reduced at the surface of the $\mathrm{Ag}_{2} \mathrm{~S}$ film. Initially the nucleus contacts the STM tip without causing a noticeable change in the current due to the large resistance of the sample.

The further evolution of the cluster radius with time, $r(t)$, is modeled as follows. We define the flux of $\mathrm{Ag}^{+}$ions, which cross the $\mathrm{Ag}_{2} \mathrm{~S} / \mathrm{Ag}$-cluster interface, that are subsequently reduced to metal atoms and added to the nucleus as $J_{\Omega_{t}}$. The flux $J_{\Omega_{t}}$ into the nucleus, therefore, corresponds to a volume change $\left(\Omega_{t}\right)$ of the nucleus according to

$$
J_{\Omega_{t}} A(r) \Omega_{a}=\frac{d \Omega_{t}(r)}{d t},
$$

where $J_{\Omega_{t}}$ is in units of atoms $/ \mathrm{m}^{2} \mathrm{~s}, A(r)$ is the crosssection area of the $\mathrm{Ag}_{2} \mathrm{~S} / \mathrm{Ag}$-cluster interface, and $\Omega_{a}$ is the volume per atom. The parameter $r$ is the radius of the cluster, which we assume for simplicity to be cylindrically symmetric.

We first solve the equations above for the case of a simplified model of tip and surface geometry. We assume an STM tip with an apex radius $R_{0}$ and smooth sample surface. We furthermore assume that the cluster grows such that it fills the space between the STM tip and the sample surface. A schematic diagram of this model system is shown in Fig. 7.

Using the parameters defined in Fig. 7, and assuming that $R_{0} \gg r$, the height of the cluster can be expressed as $h \approx \frac{r^{2}}{2 R_{0}}$ and the volume of the cluster as

$$
\Omega_{t}(r)=\frac{\pi r^{4}}{4 R_{0}} .
$$

To solve Eq. (4), let us first analyze $J_{\Omega_{t}}$. From the measurements presented in Fig. 3, we estimate that the flux of atoms into the cluster is much less than $1 \%$ of the full ion current density in $\mathrm{Ag}_{2} \mathrm{~S}$ [here, the full ion current is obtained

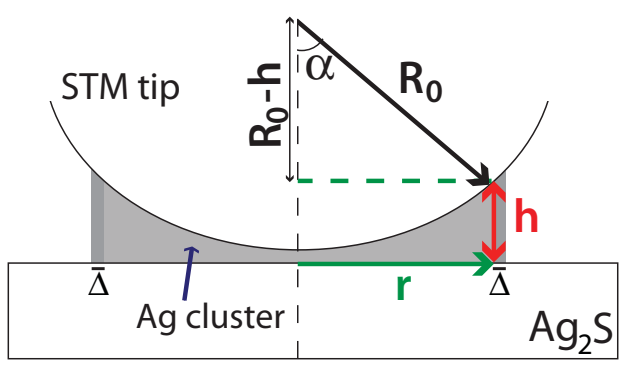

FIG. 7. (Color online) Schematic diagram of the tip and sample geometry used to model the growth of the cluster with time. $R_{0}$ is the tip radius, $r$ is the cluster radius, and $h$ is the cluster height. $\Delta$ is the width of the edge ring where the $\mathrm{Ag}$ atoms are incorporated into the nucleus. 
from the electronic current using $j_{\mathrm{Ag}^{+}}=0.1 j_{e}$ (Ref. 21)]. Therefore, when the cluster is growing, the deviation from steady state is very small, and at fixed bias voltage, after allowing for some settling, we can assume that $J_{\Omega_{t}}$ is constant over time.

Because the flux of atoms into the cluster is much smaller than the full ionic current density, we know that the atoms are restricted at the $\mathrm{Ag}_{2} \mathrm{~S} / \mathrm{Ag}$-cluster interface. This restriction is the cluster itself. The addition of extra atoms at the crosssection area just under the cluster is not likely, since the new atoms would have to work against the atoms in the cluster to displace then and accumulate in the cluster. At the edge there is no restriction. Thus, we propose that the addition of Ag to the cluster occurs only at the edge of the cluster, this edge having width $\Delta$. This forms an effective surface defined by the edge of the cluster $[2 \pi r(t)]$ and a width $\Delta$ of atomic dimensions. The parameter $A(r)$ in Eq. (4) is then given by $A(r)=2 \pi r(t) \Delta$ with $r(t)$ the radius of the cluster and $\Delta$ constant.

We fill in $\Omega_{t}(r, t)$ and $A(r, t)$ into Eq. (4), and now we only need to integrate over time. Equation (4) then results in

$$
J_{\Omega_{t}} \Omega_{a} t=\frac{r(t)^{3}}{6 R_{0} \Delta}
$$

or

$$
r(t)=\left(6 R_{0} \Delta J_{\Omega_{t}} \Omega_{a} t\right)^{1 / 3},
$$

an expression which describes the evolution of the radius $r$ of the cluster with time.

The flux of atoms into the cluster $J_{\Omega_{t}}$ is proportional to the difference of the chemical-potential gradients of $\mathrm{Ag}$ atoms and ions in the $\operatorname{Ag}_{2} \mathrm{~S}, J_{\Omega_{t}} \propto \nabla \mu_{\mathrm{Ag}}-\nabla \tilde{\mu}_{\mathrm{Ag}^{+}}$which is expected to be small. To lowest order this flux will be linear in the bias voltage $V_{s}$. Then Eq. (7) becomes

$$
r\left(V_{s}, t\right) \propto\left(R_{0} \Delta \Omega_{a} V_{s} t\right)^{1 / 3} .
$$

As we will see below, this relation describes the data in some cases very well, but not in general. We note that the power $n=1 / 3$ in Eq. (8) comes from the specific geometry for the STM tip and cluster presented in Fig. 7. In fact, Eq. (8) can be written as a general function $f$ of the time $t$ and bias voltage $V_{s}$, where $f$ generalizes the power $n$ of $r\left(V_{s}, t\right)$ defined by the specific shape of the tip and cluster. Then, in general terms,

$$
r\left(V_{s}, t\right)=f\left(\Psi V_{s} t\right)
$$

with $\Psi=R_{0} \Delta \Omega_{a}$.

Using the expression above and the dependence of the electronic current with the top contact size, we can write a scaling relation for the traces of current $I$ vs time $t$ measured at different bias voltages.

As mentioned above, the nucleus formation and growth occur at near-equilibrium conditions, i.e., near steady state, in which case the electronic current is expressed as ${ }^{12}$

$$
I\left(V_{s}\right)=K \sigma_{0} \frac{k_{B} T}{e}\left(e^{\left(e V_{s} / k_{B} T\right)}-1\right),
$$

where $k_{B}$ is Boltzmann's constant, the temperature $T=$ $295 \mathrm{~K}$, and $K=\alpha r$ describes the contact geometry, where

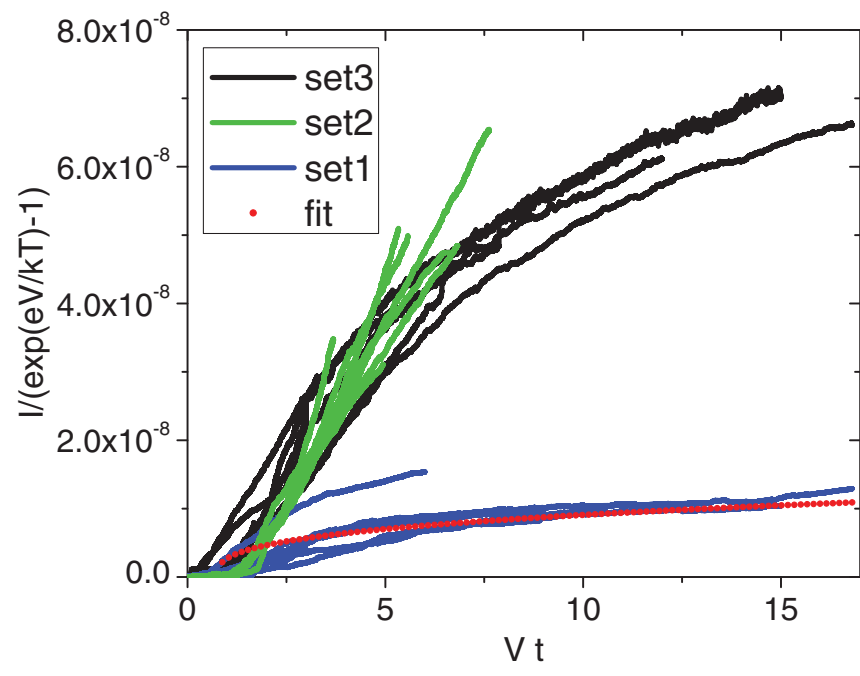

FIG. 8. (Color online) Scaling of three sets of data, measured at different positions on the sample and with different STM tips. The red dotted line is a fit to one of the traces from set 1 with a power law of $V_{s} t$ of $1 / 3$, as the result obtained with our simplified model [Eq. (8)].

$\alpha$ is a constant of order unity and depends on the size and shape of the top contact. In our case, the top contact is the Ag cluster that is growing at the surface, therefore, $r=r\left(V_{s}, t\right)$.

From Eqs. (9) and (10), we obtain the final form,

$$
I\left(V_{s}, t\right)=\Gamma f\left(\Psi V_{s} t\right)\left(e^{\left(e V_{s} / k_{B} T\right)}-1\right),
$$

with $\Gamma=\alpha \sigma_{0} \frac{k_{B} T}{e}$.

Equation (11) predicts that if we plot $I\left(V_{s}, t\right) /$ $\left(e^{\left(e V_{s} / k_{B} T\right)}-1\right)$ vs $V_{s} t$, for one set of data of current vs time traces, for example, the set presented in Fig. 3(b), the data should collapse onto a single curve. This is confirmed in Fig. 8 for three different sets of data. Each set of data contains traces of current vs time, measured at voltages from +20 to +60 $\mathrm{mV}$. Each set of data was measured with a different tip and at a different position on the sample, resulting in a different function $f$ and/or proportionally constant $\Gamma$. For example, the data set 1 was measured with an etched STM tip with a well-defined shape, and the data of sets 2 and 3 were measured with a manually cut tip of which the geometry is not well defined. This can explain variations in the power law and the fact that the best scaling with a power law given by Eq. (8) is obtained for the etched tip (set 1).

\section{CONCLUSION}

We have observed the occurrence of two physical processes in $\mathrm{Ag}_{2} \mathrm{~S}$ under the influence of the applied bias voltage: nucleation of a $\mathrm{Ag}$ cluster on the surface, and at higher bias, nucleation of a wire inside the $\mathrm{Ag}_{2} \mathrm{~S}$ film. We have found that surface nucleation is possible at very low bias voltages, below $20 \mathrm{mV}$. Nucleation of a nanowire inside the film occurs at bias voltages higher than $70 \mathrm{mV}$ at room temperature. At this point it is not possible to decide whether the nucleation is governed strictly by the bias 
voltage alone, or by the electric field that it causes, as was recently demonstrated for amorphous-crystalline phase change materials. ${ }^{9,22,23}$

$\mathrm{Ag}_{2} \mathrm{~S}$ forms an interesting model system for the understanding of memristive nanoionic devices. In nanoionic devices, the ion transport properties play a significant role in the determination of retention times, cycling endurance, and writing and reading voltages. The type of measurements presented here are important to screen and select future candidate materials and processes for memory-resistive devices.

\section{ACKNOWLEDGMENTS}

We thank H. D. Wiemhöfer for his interest in this project and for discussions. We thank Ruud Tromp for making the UHV-STM system available for this work, Federica Galli for technical support on the STM, and Jelmer Wagenaar for discussions. M.M.-M. is grateful to Alpana Nayak for support and discussions in the extended visit to Tsukuba. This work is part of the research program of the Dutch Foundation for Fundamental Research on Matter (FOM) that is financially supported by NWO. *morales@physics.leidenuniv.nl

${ }^{1}$ M. Wuttig and N. Yamada, Nat. Mater. 6, 824 (2007).

${ }^{2}$ D. Lencer, M. Salinga, B. Grabowski, T. Hickel, J. Neugebauer, and M. Wuttig, Nat. Mater. 7, 972 (2008).

${ }^{3}$ R. Waser and M. Aono, Nat. Mater. 6, 833 (2007).

${ }^{4}$ R. Waser, R. Dittmann, G. Staikov, and K. Szot, Adv. Mater. 21, 2632 (2009).

${ }^{5}$ T. Hino, T. Hasegawa, K. Terabe, T. Tsuruoka, A. Nayak, T. Ohno, and M. Aono, Sci. Technol. Adv. Mater. 12, 013003 (2011).

${ }^{6}$ M. N. Kozicki and M. Mitkova, Nanotechnology (Wiley-VCH Verlag GmbH \& Co. KGaA, 2010).

${ }^{7}$ A. Geresdi, A. Halbritter, A. Gyenis, P. Makk, and G. Mihaly, Nanoscale 3, 1504 (2011).

${ }^{8}$ A. Nayak, T. Tamura, T. Tsuruoka, K. Terabe, S. Hosaka, T. Hasegawa, and M. Aono, J. Phys. Chem. Lett. 1, 604 (2010).

${ }^{9}$ V. G. Karpov, Y. A. Kryukov, I. V. Karpov, and M. Mitra, Phys. Rev. B 78, 052201 (2008).

${ }^{10}$ T. Tamura, T. Hasegawa, K. Terabe, T. Nakayama, T. Sakamoto, T. Sunamura, H. Kawaura, S. Hosaka, and M. Aono, J. Phys.: Conf. Ser. 61, 1157 (2007).

${ }^{11}$ M. Morales-Masis, S. J. van der Molen, W. T. Fu, M. B. Hesselberth, and J. M. van Ruitenbeek, Nanotechnology 20, 095710 (2009).
${ }^{12}$ M. Morales-Masis, H. D. Wiemhofer, and J. M. van Ruitenbeek, Nanoscale 2, 2275 (2010).

${ }^{13}$ S. Kashida, N. Watanabe, T. Hasegawa, H. Iida, M. Mori, and S. Savrasov, Solid State Ionics 158, 167 (2003).

${ }^{14}$ Z. C. Wang, T. K. Gu, T. Kadohira, T. Tada, and S. Watanabe, J. Chem. Phys. 128, 014704 (2008).

${ }^{15}$ M. H. Hebb, J. Chem. Phys. 20, 185 (1952).

${ }^{16}$ C. Wagner, J. Chem. Phys. 21, 1819 (1953).

${ }^{17}$ K. Terabe, T. Nakayama, T. Hasegawa, and M. Aono, J. Appl. Phys. 91, 10110 (2002).

${ }^{18}$ Z. Xu, Y. Bando, W. L. Wang, X. D. Bai, and D. Golberg, ACS Nano 4, 2515 (2010).

${ }^{19}$ H. Schmalzried, Prog. Solid State Chem. 13, 119 (1980).

${ }^{20}$ H. Rickert and H. D. Wiemhöfer, Ber. Bunsenges. Phys. Chem. 87, 236 (1983).

${ }^{21}$ U. Bonnecaze, A. Lichanot, and S. Gromb, J. Phys. Chem. Solids 39, 299 (1978).

${ }^{22}$ I. V. Karpov, M. Mitra, D. Kau, G. Spadini, Y. A. Kryukov, and V. G. Karpov, Appl. Phys. Lett. 92, 173501 (2008).

${ }^{23}$ D. Krebs, S. Raoux, C. T. Rettner, G. W. Burr, M. Salinga, and M. Wuttig, Appl. Phys. Lett. 95, 082101 (2009).

${ }^{24}$ This value depends on the nonstoichiometry of $\mathrm{Ag}_{2+\delta} \mathrm{S}$. The value of $70 \mathrm{mV}$ applies for a sample for which the Ag cation concentration is fixed by an intimate contact to a $\mathrm{Ag}$ metal reservoir. 